PSFC/JA-03-1

Design and Emission Uniformity Studies of a 1.5-MW Gyrotron Electron Gun

Anderson, J. P., Korbly, S. E., Temkin, R. J., Shapiro, M. A., Felch, K. L.*,

Cauffman, S.*

December 2002

Plasma Science and Fusion Center

Massachusetts Institute of Technology

Cambridge, MA 02139 USA

\author{
* Microwave Power Products Division \\ Communications and Power Industries \\ Palo Alto, CA 94303 USA
}

This work was supported by the U.S. Department of Energy, Office of Fusion Sciences and Virtual Laboratory for Technology. Reproduction, translation, publication, use and disposal, in whole or in part, by or for the United States government is permitted.

Submitted for publication to IEEE Transactions on Plasma Science. 


\title{
Design and Emission Uniformity Studies of a 1.5 MW Gyrotron Electron Gun
}

\author{
James P. Anderson ${ }^{1}$, Stephen E. Korbly ${ }^{1}$, Richard J. Temkin ${ }^{1}$, Michael A. Shapiro ${ }^{1}$, \\ K. L. Felch ${ }^{2}$, and S. Cauffman ${ }^{2}$ \\ ${ }^{1}$ Plasma Science and Fusion Center, Massachusetts Institute of Technology, \\ Cambridge, MA 02139 \\ ${ }^{2}$ Microwave Power Products Division, Communications and Power Industries, \\ Palo Alto, CA 94303
}

\begin{abstract}
We present the design and initial operation of a $96 \mathrm{kV}, 40 \mathrm{~A}$ Magnetron Injection Gun (MIG) for a $1.5 \mathrm{MW}, 110$ $\mathrm{GHz}$ gyrotron. A critical parameter for the successful application of this electron gun is the uniformity of electron emission. The I-V curve of emission, at a series of temperatures, is measured. Analysis indicates that the work function of the emitter is $1.6 \mathrm{eV}$ with a (total) spread of $0.07+/-0.01 \mathrm{eV}$. Measurement of the azimuthal emission uniformity with a rotating probe indicates that the work function variation around the azimuth, the global spread, is $0.04+/-0.02$ $\mathrm{eV}$. The spread due to local (microscopic scale) work function variations is then calculated to be $0.06+/-0.02 \mathrm{eV}$. Temperature variation can be ruled out as the cause of the observed emission nonuniformity.
\end{abstract}

\section{INTRODUCTION}

Presently, long pulse and continuous wave gyrotrons operating at $1 \mathrm{MW}$ in the 110-170 GHz range are under development [1][2] [3][4][5][6]. The $1 \mathrm{MW}$ power level is adequate for existing electron cyclotron heating (ECH) systems, but future systems using 10 to $50 \mathrm{MW}$ of total power will require more power per tube in order to be cost effective. At MIT, we are studying a $1.5 \mathrm{MW}, 110 \mathrm{GHz}$ gyrotron. Our goal is to demonstrate stable, single mode operation with an efficiency greater than $50 \%$. Our plan is to scale to higher powers the $110 \mathrm{GHz}$ gyrotron produced by Communications and Power Industries (CPI), Palo Alto, CA, that successfully produced $1 \mathrm{MW}$ [2]. Two new components that will be incorporated into the $1.5 \mathrm{MW}$ tube are a depressed collector and, as is discussed in this paper, a $96 \mathrm{kV}, 40 \mathrm{~A}$ diode magnetron injection electron gun (MIG). In order to better understand the unique features of this gyrotron, including the role of trapped and reflected electrons, a short pulse (3 $\mu \mathrm{s})$ prototype will be built and operated at MIT. The first experiments will have a grounded collector and axial output coupling (Fig. 1). Later experiments will use an internal mode converter and a depressed collector.

One of the main issues of concern which arises during gyrotron operation is the emission uniformity of the electron gun. Recent studies indicate that unwanted mode competition can be generated in a gyrotron due to nonuniform emission of electrons from the thermionic cathode used in MIG electron guns [7][8]. This mode competition significantly decreases the microwave efficiency of the device. Nonuniform emission also leads to nonuniform heating or hot spots in the collector which may cause excessive outgassing or even melting. Therefore, to reduce mode competition and improve gyrotron performance, a good understanding of the uniformity of emission from the cathode surface is necessary.

One previous investigation of nonuniform cathode emission in a gyrotron was motivated by the observation of a large-scale azimuthal emission asymmetry in the electron beam. The cathode had a partial failure of its heater resulting in emission of only a half-circle of beam. This resulted in a large reduction in gyrotron efficiency [9]. That gun was rebuilt and an improved efficiency was obtained. Later studies of gyrotron emission uniformity at MIT indicated that even in the presence of good thermal uniformity, cathode emission was still nonuniform [10]. Studies of the power distribution in the collector of megawatt power level gyrotrons also indicate an azimuthal asymmetry. Recent 


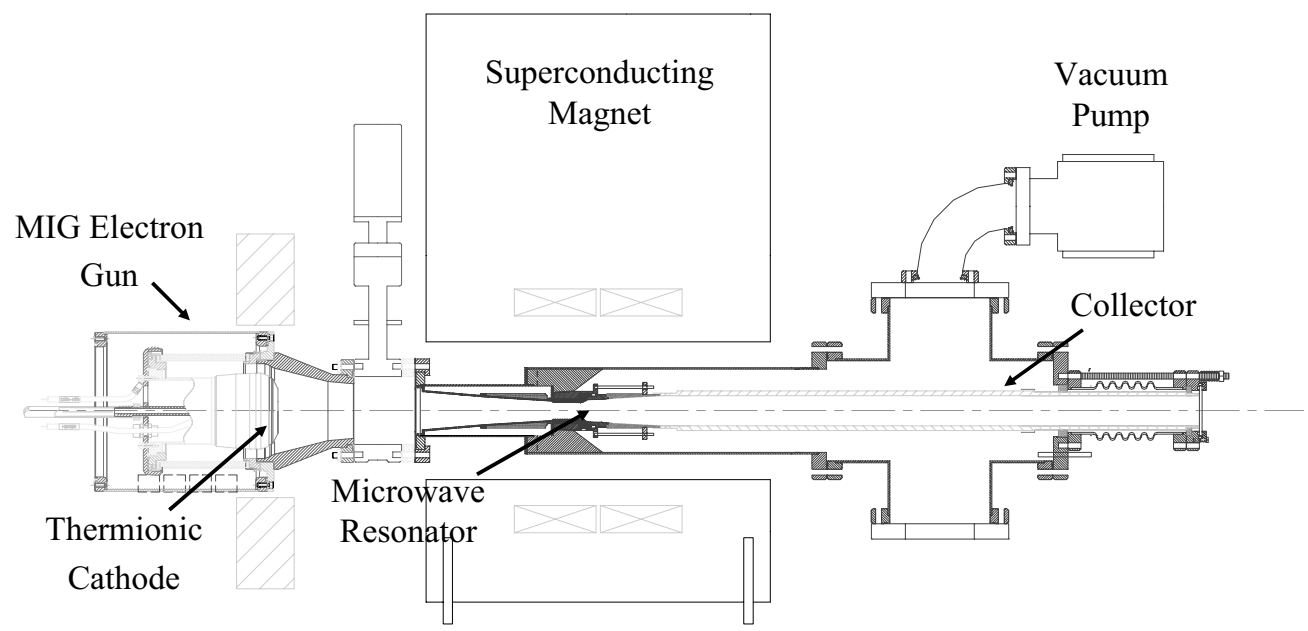

Fig. 1. The schematic of the initial 1.5 MW gyrotron experiment at MIT with axial output coupling. Included in the figure are the electron gun, the microwave resonating structure, and the electron beam collector.

research by Glyavin et al. [11] and Advani et al. [7] have identified work function variation as an issue in gyrotron cathode emission uniformity.

In this paper, we focus on the design of the electron gun which will be used for the gyrotron experiments. In addition, following a brief discussion of the theory behind electron emission from a thermionic cathode, preliminary measurements are presented of the electron beam emission uniformity produced from the gun which was fabricated based on this design. The results of these measurements are then compared with those of similar cathodes.

\section{Electron Gun Design}

The 1.5 MW, $110 \mathrm{GHz}$ gyrotron design began with a parametric study in order to identify the key gyrotron features. We considered a variety of constraints including cavity ohmic losses, mode competition, and power supply limitations on the beam voltage and current. The design is based on a tapered cylindrical cavity operating in the $\mathrm{TE}_{22,6,1}$ mode. This mode, which is the same mode used in the $1 \mathrm{MW}$ CPI tube, will provide acceptable ohmic losses even at the $1.5 \mathrm{MW}$ level using a redesigned cavity. We anticipate no mode competition problems if the cavity length is properly chosen. Past experiments at MIT [12], IAP (N. Novgorod, Russia) [13], and FZK (Karlsruhe, Germany) [14] have shown that $1 \mathrm{MW}[13]$ and $1.5 \mathrm{MW}$ [12][14] power levels can be generated in extremely high order modes with good efficiency and minimal problems from competing modes. The single-stage depressed collector will be at ground potential for safety reasons, while the cavity region will be operated at a positive high voltage. The voltage difference $\mathrm{V}_{D E P}$ between the cavity and the collector represents the beam energy that is recovered.

An important design parameter is the beam velocity ratio, $\alpha$, which is defined as the ratio of the transverse velocity $v_{\perp}$, to the parallel velocity $v_{\|}$, or $v_{\perp} / v_{\|}$. Typically a high ratio is desirable for efficient operation, but this can lead to trapped electrons between the collector and gun regions that can degrade performance. Since most of the parallel electron energy can be recovered, it is actually preferable to operate at lower $\alpha$ to avoid trapped electrons. The expected total efficiency is shown in Fig. 2 for the design velocity ratio, $\alpha=1.4$. Although higher efficiencies are possible at higher $\alpha$, this also requires operating closer to the limiting current. The design parameters are listed in Table I. Using the $1 \mathrm{MW}$ CPI gun cathode geometry as a starting point, the gun design was completed using EGUN [15]. The electron gun geometry and beam trajectory are shown in Fig. 3. A typical simulation of the velocity ratio and perpendicular velocity spread is shown in Fig. 4 where an $\alpha=1.4$ produces a velocity spread of $2.5 \%\left(\delta v_{\perp} / v_{\perp}=0.025\right)$ due to beam optics. Additional sources of velocity spread include $4.2 \%$ from surface roughness, $0.9 \%$ from machining or misalignment errors, 


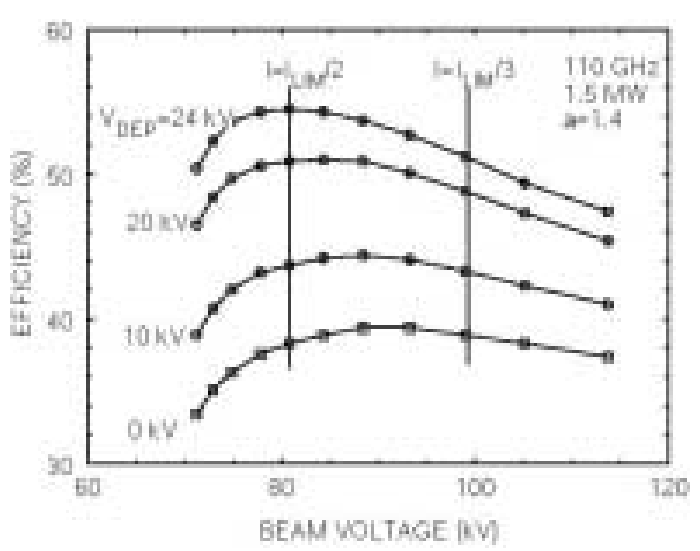

Fig. 2. Total efficiency for a $1.5 \mathrm{MW}, 110 \mathrm{GHz}$ gyrotron with a velocity ratio of 1.4..

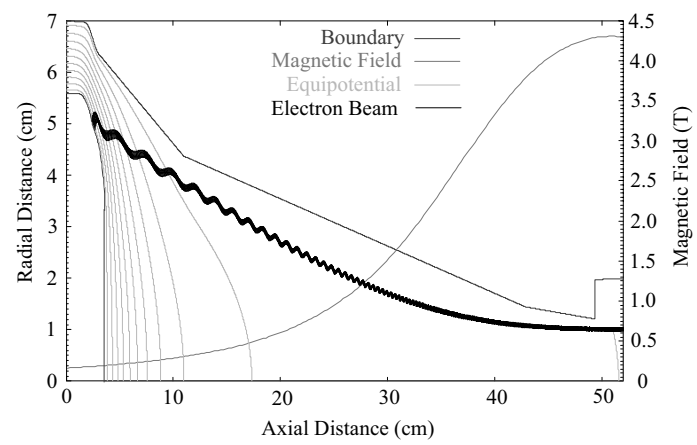

Fig. 3. Geometry, beam trajectory, equipotenials and magnetic field for the $110 \mathrm{GHz}$ diode gun design.

and $0.6 \%$ from thermal spread and nonuniform emission [12][16]. EGUN simulations were performed to verify the effect on the velocity spread due to mechanical misalignment, machining errors, voltage ripple, magnetic field inhomogeneities, and current nonuniformity. As a result, the final beam spread is predicted to be about $5 \%$.

\section{EMISSION UNIFORMity THEORY}

In this section, we discuss the theory behind nonuniform emission by first describing the well-known idealized current laws [17][18][19], and then showing how these equations are transformed in the more realistic case, when there is a spread in the work function. Moreover, we make the differentiation between the local and global amounts of work function spread observed in thermionic cathodes.

\begin{tabular}{|c|c|}
\hline Frequency & $110 \mathrm{GHz}$ \\
\hline Output Power & $1.5 \mathrm{MW}$ \\
\hline Beam Voltage & $96 \mathrm{kV}$ \\
\hline Beam Current & $40 \mathrm{~A}$ \\
\hline Mode & $\mathrm{TE}_{22,6}$ \\
\hline Velocity Ratio & 1.4 \\
\hline Velocity Spread & 2.5 \\
\hline Cavity B Field & $4.3 \mathrm{~T}$ \\
\hline Compression & 22.13 \\
\hline
\end{tabular}

TABLE I

1.5 MW, $110 \mathrm{GHz}$ gyrotron MIG electron gun design. 


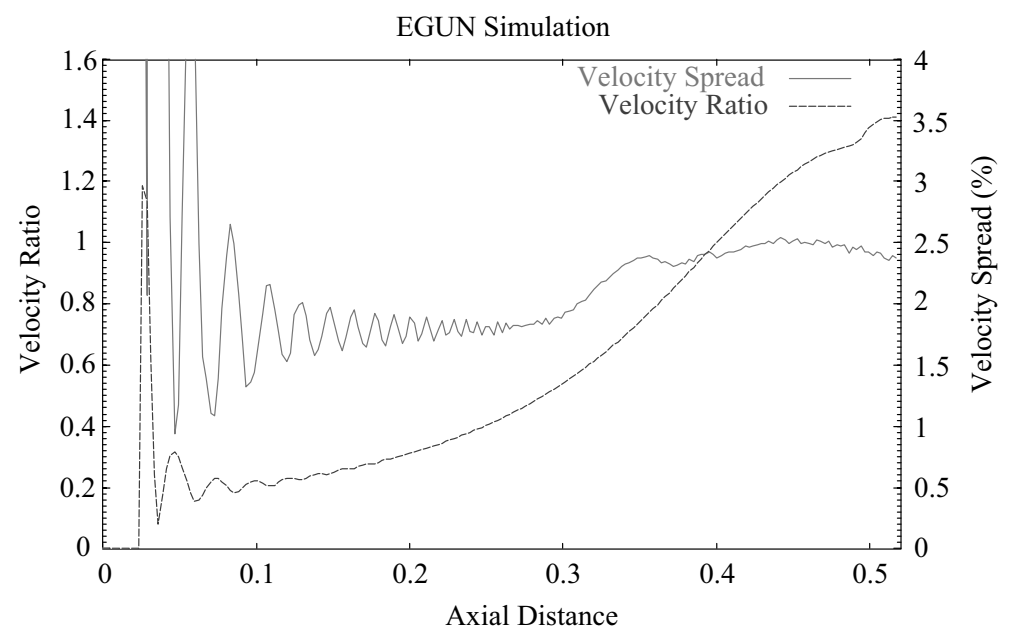

Fig. 4. Velocity ratio and perpendicular velocity spread as a function of axial distance.

The ideal annular cathode has no variations in work function or temperature. For this case, there is an abrupt transition between space-charge limited electron emission at lower voltages, and temperature limited emission. The current density in the space-charge limited region is governed by an equation derived from the Child-Langmuir law for a diode [20]:

$$
J_{S C L}=\kappa V^{3 / 2} \quad V \leq V_{T}
$$

where the constant $\kappa$ is defined as the perveance of the cathode and depends on the cathode geometry. Past a threshold voltage, $V_{T}$, the emitted current density follows the Richardson-Dushman equation in the temperature limited region:

$$
J_{T L}=A_{o} T^{2} \exp \left[\frac{-e}{k T}\left(\phi-\sqrt{\frac{e E}{4 \pi \varepsilon_{o}}}\right)\right] \quad V>V_{T}
$$

where $\phi$ is the work function and $E$ is the electric field which contributes to the Schottky effect, $T$ is the temperature of the cathode, $k$ is Boltzmann's constant, and the constant $A_{o}=120 \mathrm{~A} / \mathrm{cm}^{2} \mathrm{deg}^{2}$. The threshold voltage, where $J_{S C L}=J_{T L}$, is determined by the work function and temperature of the cathode. If the total current density, $J_{\text {Total }}$, is plotted for increasing voltage, the current linearly increases as $V^{3 / 2}$ until the threshold voltage ( $V_{T}$ in Fig. 5for the single work function curve) is reached. Past this voltage, the current is saturated and becomes nearly constant. There is a small slope due to the Schottky effect.

In reality, however, a cathode has a spread in the work function and temperature. Therefore, electron emission varies from one particular location of the cathode to another. In this case, there is a smooth transition between space-charge limited operation and temperature limited operation (Fig. 5). The spread in the work function may be determined by examining current behavior in this transition region. Conversely, the total current at a given voltage is determined from a known work function distribution by summing up the portion of the cathode which is still emitting in the space-charge limited region and the cathode region which is temperature limited. Mathematically this may be stated as:

$$
J_{V}=\int_{\phi_{\min }}^{\phi_{V}} J_{S C L} D(\phi) d \phi+\int_{\phi_{V}}^{\phi_{\max }} J_{T L} D(\phi) d \phi
$$

where $D(\phi)$ is the normalized work function distribution, and $\phi_{V}$ is defined as:

$$
\phi_{V}=\sqrt{\frac{e E}{4 \pi \varepsilon_{o}}}-\frac{k T}{e} \ln \left(\frac{\kappa V^{3 / 2}}{A_{o} T^{2}}\right)
$$




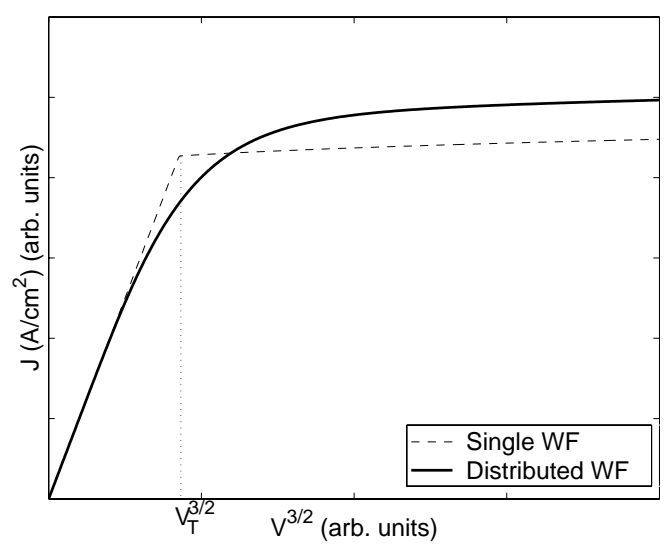

Fig. 5. The I-V characteristic for an ideal cathode with a single work function value (dashed line) has an abrupt transition at $V_{T}^{3 / 2}$. The cathode with a Gaussian distributed work function shows a smooth transition between space-charge limited operation and temperature limited.

As an example, for a cathode with a Gaussian work function distribution centered at $\phi_{o}$ with standard deviation $\sigma$, the current density is given by the following equation [21]:

$$
\begin{gathered}
J_{V}=\frac{\kappa V^{3 / 2}}{2}\left[1+\operatorname{erf}\left(\frac{\phi_{T}-\phi_{o}}{\sigma \sqrt{2}}\right)\right]+ \\
A_{o} T^{2}\left[1-\operatorname{erf}\left(\frac{\phi_{T}-\phi_{o}+\frac{e \sigma^{2}}{k T}}{\sigma \sqrt{2}}\right)\right] \times \\
\exp \left[\frac{-e}{k T}\left(\phi_{o}-\sqrt{\frac{e E}{4 \pi \varepsilon_{o}}}-\frac{e \sigma^{2}}{2 k T}\right)\right]
\end{gathered}
$$

where $\phi_{T}$ is $\phi\left(V_{T}\right)$, or the transitional work function, as illustrated in Fig. 5.

In this research, we analyze the I-V curves by assuming that the work function distribution may be characterized as a Gaussian with central value $\phi_{o}$ and standard deviation $\sigma$, which we refer to as the work function spread. Eq. 5 will be used to fit the measured data to determine these two free parameters [21].

In addition, a distinction may be made between the global and local work function spreads. It is known that small areas of the cathode (as small as $500 \mu \mathrm{m}$ on a side) show an effect due to work function variation [22]. We denote this variation as a "local" spread in work function, meaning that it occurs over a microscopic area of the emitter (Fig. 6). In addition to the local spread, different regions of the emitter, separated on a large scale length of order centimeters, may have a different local mean value of the work function. We denote this variation to be the "global" spread in work function. We assume that the local and the global spreads are uncorrelated. In that case:

$$
\sigma_{\text {Total }}^{2}=\sigma_{\text {global }}^{2}+\sigma_{\text {local }}^{2}
$$

One method of determining the local spread is by taking current measurements over very small locations of the cathode [22][23]. Another method, such as the one employed at MIT, determines the global spread first, from which the local spread may be calculated using the total spread and Eq. 6 .

\section{Emission Uniformity Measurements}

According to previous emission studies [21], a Gaussian distribution is a reasonable approximation for the work function of most thermionic cathodes. Therefore, a Gaussian distribution is assumed for the cathode analyzed at MIT. In the first step, the electron beam current is measured at several 


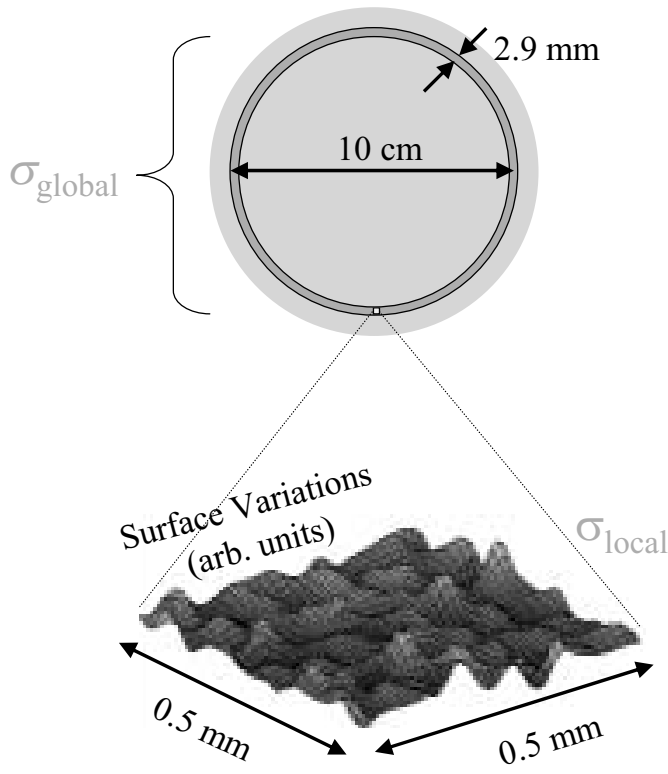

Fig. 6. The annular cathode (top) has a much larger area than sections which contribute to the local spread (bottom). An example of surface spatial variations over a small section of the cathode ( $500 \mu \mathrm{m}$ on a side), which give rise to a local spread in the work function, is shown in the bottom figure. The current variations are on a similar spatial scale.

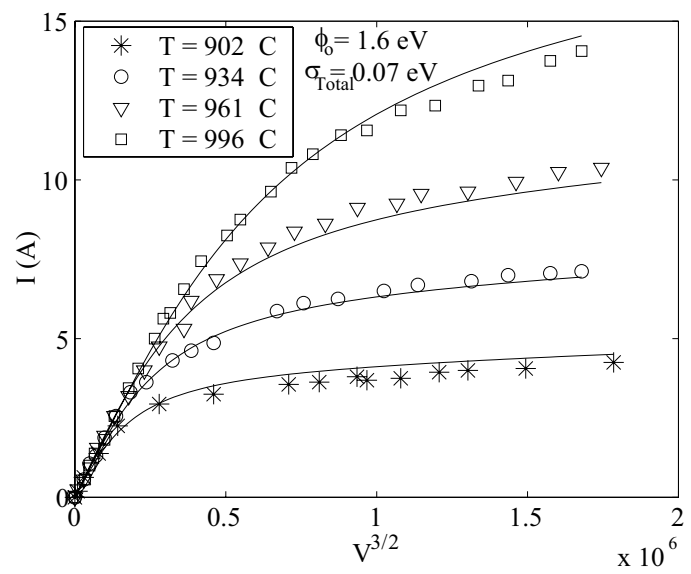

Fig. 7. The current-voltage data is taken for various cathode temperatures. The current measured at each voltage point is accurate to within $1 \%$. The best Gaussian work function fit to the measured I-V data taken at various temperatures is when the center work function value is $\phi_{o}=1.6 \mathrm{eV}$, and the work function spread is $\sigma_{\text {Total }}=0.07$ $\mathrm{eV}$.

different cathode temperatures over a wide range of voltages (Fig. 7). Next, the data are fit using Eq. 5, which yields a form for the total current in terms of the Gaussian distribution parameters, $\phi_{o}$, and $\sigma$. For the I-V data shown in Fig. 7, the work function center was found to be $\phi_{o}=1.6 \mathrm{eV}$, with a deviation of $\sigma=\sigma_{\text {Total }}=0.07 \pm 0.01 \mathrm{eV}$. Since the current measurements were taken for the entire beam, this deviation represents the total spread in the work function, $\sigma_{\text {Total }}$. Note that these values provide a reasonably good fit at all temperatures.

To determine the global spread, detailed measurements of the cathode emission around the azimuth must be taken. Therefore the emitted electron beam is examined at many different azimuthal locations using a rotating current probe as part of the beam collector (the far right end of the collector shown in Fig. 1.) The setup of this diagnostic tool is shown in Fig. 8. Most of the annular beam enters a metal cylinder and impacts on the inner wall of the structure as the beam expands in a region of decreasing magnetic field. Some of the beam, however, passes through a narrow $\left(10^{\circ}\right)$ slot, from which 


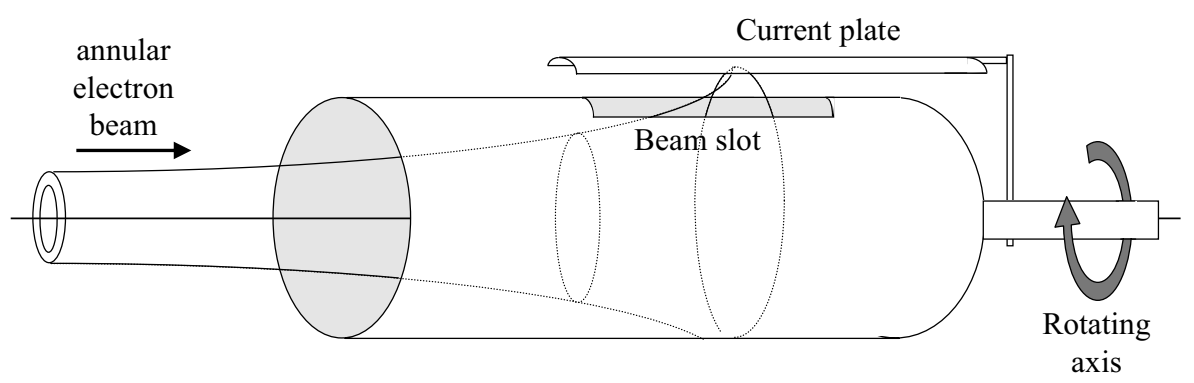

Fig. 8. The diagnostic used for measuring the beam current at a particular azimuthal angle consists of a conducting tube, which the electron beam enters, and a narrow beam slot, through which a small angular section of the beam exits. This portion of the beam current is measured by a current plate, placed above the beam slot. The structure is rotated around the azimuth to sample the beam current at various angles.

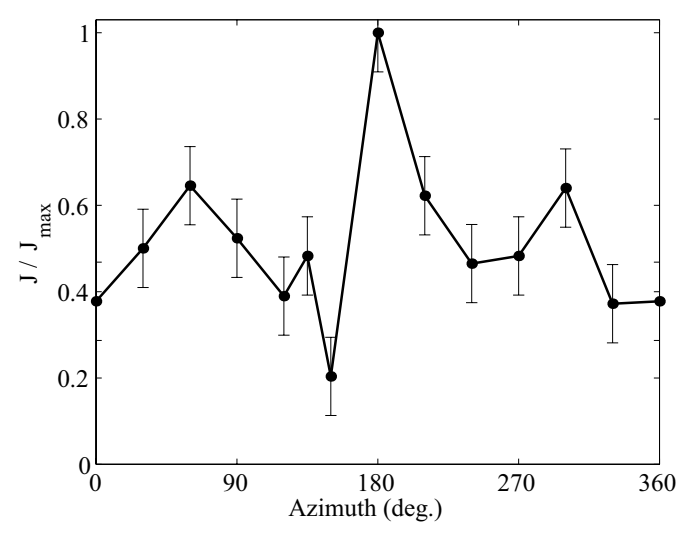

Fig. 9. The azimuthal current density scan of the emitted beam.

the current is sampled. The probe is rotated to measure the current at many different angles around the gyrotron axis. A normalized scan taken of the beam emitted from the cathode used at MIT is plotted in Fig. 9.

The global work function spread is determined from this normalized scan by first noting that each data point represents a ratio of the current density at that location to a maximum current value, or $J / J_{\max }$. Since the temperature-limited current in the Richardson-Dushman equation (Eq. 2) has an $\exp (-e \phi / k T)$ dependence, the maximum current density, $J_{\max }$, occurs at the location where the work function is smallest, $\phi_{\min }$. The ratio of the two currents is related to the work function:

$$
\frac{J}{J_{\max }}=\exp \left[\frac{-e\left(\phi-\phi_{\min }\right)}{k T}\right]
$$

This may be rewritten such that it is possible to determine the work function difference from the measured ratio:

$$
\phi-\phi_{\min }=-\frac{k T}{e} \ln \left(\frac{J}{J_{\max }}\right)
$$

Using this equation, the normalized current values, such as those shown in Fig. 9, may be converted to work function differences. The distribution of these work function differences are shown in Fig. 10. If we assume a Gaussian distribution, the standard deviation of $D\left(\phi-\phi_{\min }\right)$ is the same as $D(\phi)$. This deviation represents the global work function spread, $\sigma_{\text {global }}$. For the distribution shown in Fig. 10 , the global work function spread is $\sigma_{\text {global }}=0.04 \pm 0.02 \mathrm{eV}$. This value of $\sigma_{\text {global }}$ is not surprising since the standard deviation of $\phi$ in Eq. 7 must be comparatively smaller than $k T$ (which is $0.11 \mathrm{eV}$ at $T=1000^{\circ} \mathrm{C}$ ) to produce the variations in the current seen in Fig. 9. 


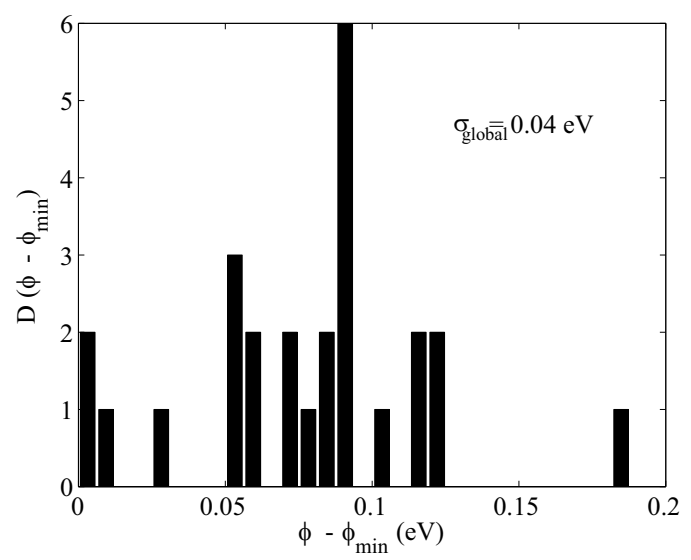

Fig. 10. The distribution of work function differences is determined from the normalized current values in Fig. 9. If a Gaussian distribution is assumed, then $\sigma_{\text {global }}=0.04 \mathrm{eV}$.

To this point we have assumed that current emission variations are due to work function spread and not temperature spread. We can justify this assumption by noting what the value of the temperature spread would have to be in order to cause the same type of azimuthal current variations seen by the current probe. For any two data points, if we assume the work function was identical at each location but the temperature varies, we find:

$$
\frac{J_{1}}{J_{2}}=\exp \left[\frac{-e \phi}{k}\left(\frac{1}{T_{1}}-\frac{1}{T_{2}}\right)\right]
$$

For the current data shown in Fig. 9, the maximum current density variation is $J_{1} / J_{2}=5.2$. Assuming a nominal work function value of $1.6 \mathrm{eV}$ and a temperature of around $1000^{\circ} \mathrm{C}$, then the temperature difference to cause this much variation in the current would have to be at least $84^{\circ} \mathrm{C}$. Since measurements of the cathode indicate a temperature uniformity of better than $\pm 5^{\circ} \mathrm{C}$, the dominant cause of the current variations is not temperature spread, but work function spread.

Since the total work function spread is determined from the current density data shown in Fig. 7, and the global spread is determined from the current probe measurements plotted in Fig. 9, the local spread may be calculated by applying Eq. 6. Using the numbers for the cathode at MIT, the local work function spread is $\sigma_{\text {local }}=0.06 \pm 0.02 \mathrm{eV}$. The observed local work function spread may be the result of several effects. One possibility is that the emitter material consists of small crystals whose surfaces have different crystal orientations. Since the work function depends on the crystal orientation, a spread in work function would arise [20]. A second possibility is that surface roughness gives rise to a variation in the effective electric field strength at the emitter surface. Estimates of the variation in electric field have been previously carried out in analyzing the origin of velocity spread in electron guns [24]. The variation can be large because of the presence of points on the emitter surface; a typical value of the local electric field variation is $E_{\max }=4 E_{\min }$. Using this result in Eq. 2 yields an estimated variation in emission current density, through the Schottky effect, of $J_{1} / J_{2}=2.2$. This current variation is not due to a variation in work function; however, it is the same size as the variation caused by a work function rms spread of about $0.03 \mathrm{eV}$. This spread is smaller than the measured value but is not negligible. There may also be other effects that cause the observed variation in local work function spread.

Results for the MIT cathode are summarized in Table II. Research on similar cathodes has produced comparable values for work function spreads. For example, the local work function spread of a scandate cathode measured in [22] was $0.03 \mathrm{eV}$. In [21], the total work function spread of an M-type tungsten cathode was $0.05 \mathrm{eV}$. Finally, in [23], the total work function spread of a large thermionic cathode was $0.08 \mathrm{eV}$, while the local spread was around $0.06 \mathrm{eV}$. 


\begin{tabular}{|c|c|c|}
\hline$\sigma_{\text {Total }}$ & $\sigma_{\text {global }}$ & $\sigma_{\text {local }}$ \\
\hline $0.07 \pm 0.01 \mathrm{eV}$ & $0.04 \pm 0.02 \mathrm{eV}$ & $0.06 \pm 0.02 \mathrm{eV}$ \\
\hline
\end{tabular}

TABLE II

Summary of work function spreads from beam measurements for the gyrotron cathode.

\section{Conclusion}

We have presented the design of a $96 \mathrm{kV}, 40 \mathrm{~A}$ electron gun which is designed to produce $1.5 \mathrm{MW}$ of microwave power at $110 \mathrm{GHz}$ in gyrotron experiments at MIT. The design is based on previous gyrotron tube designs.

In addition to the gun design, the theory behind electron emission from a thermionic cathode has been presented. The current density produced from an ideal emitter is described by two basic equations (Eq. 1 and Eq. 2) and is dependent on the voltage, work function, and temperature of the cathode. When the work function is distributed these equations are combined into one equation (Eq. 3). The emission quality of the cathode is determined from the spread of the distribution. Both local and global effects contribute to the total work function spread.

The work function distribution has been estimated from measurements of the cathode used in the gun constructed from the design. Specifically, the total work function spread was calculated from fitting the current density data with Eq. 5, which assumes a Gaussian distribution for the work function. Next, a current probe was used to determine the global spread and, by applying Eq. 6, the local spread was determined. Future research will attempt to determine the origin of the observed global work function spread, which is undesirable for gyrotron operation.

\section{ACKNOWLEDGMENT}

The authors wish to acknowledge Clifford Fortgang of Los Alamos and Lou Falce of CPI for helpful discussions.

\section{REFERENCES}

[1] T. Imai, N. Kobayashi, R. Temkin, M. Thumm, M. Q. Tran, V. Alikaev, "ITER R\&D: auxiliary systems: electron cyclotron heating and current drive system," Fusion Engineering and Design, vol. 55, no. 2-3, pp. 281-9, 2001.

[2] T.S. Chu, M. Blank, P. Borchard, P. Cahalan, S. Cauffman, K. Felch, H. Jory, G. Saraph, D. Wagner, "Recent progress in producing megawatt gyrotrons for ECH applications," 25th International Conference on Infrared and Millimeter Waves (Cat. No. 00EX442). IEEE, Piscataway, NJ, USA, pp. 13-14, 2000.

[3] S. Alberti, A. Arnold, E. Borie, G. Dammertz, V. Erckmann, P. Garin, E. Giguet, S. Illy, G. Le-Cloarec, Y. Le-Goff, R. Magne, G. Michel, B. Piosczyk, C. Tran, M.Q. Tran, M. Thumm, D. Wagner, "European high-power CW gyrotron development for ECRH systems," Fusion Engineering and Design, vol. 53, pp. 387-97, 2001.

[4] A. Kasugai, K. Sakamoto, K. Takahashi, K. Kajiwara, H. Shoyama, Y. Ikeda, M. Tsuneoka, T. Fujii, T. Kariya, Y. Mitsunaka, T. Imai, "1 MW and long pulse operation of gaussian beam output gyrotron with CVD diamond window for fusion devices," Fusion Engineering and Design, vol. 53, pp. 399-406, 2001.

[5] V.E. Zapevalov, G.G. Denisov, V.A. Flyagin, A.S. Fix, A.N. Kuftin, A.G. Litvak, M.V. Agapova, V.N. Iljin, V.A. Khmara, V.E. Myasnikov, V.O. Nichiporenko, L.G. Popov, S.V. Usachev, V.V. Slikaev, V.I. Iljin, "Development of $170 \mathrm{GHz} / 1 \mathrm{MW}$ Russian gyrotron for ITER," Fusion Engineering and Design, vol. 53, pp. 377-85, 2001.

[6] K. Kreischer, S. Korbly, M. Shapiro, R.J. Temkin, S. Cauffman, K. Felch, "The development of a 1.5 MW, 110 GHz gyrotron for plasma heating," Internation Vacuum Electronics Conference 2000 (Cat. No. 00EX392). IEEE, Piscataway, NJ, USA, paper 18.4, 2000.

[7] R. Advani, J.P. Hogge, K.E. Kreischer, M. Pedrozzi, M.E. Read, J.R. Sirigiri, R.J. Temkin, "Experimental investigation of a 140-GHz coaxial gyrotron oscillator," IEEE Trans. Plasma Sci., vol. 29, no. 6, pp. 943-50.

[8] G. S. Nusinovich, A. N. Vlasov, M. Botton, T. M. Antonsen, Jr., S. Cauffman, and K. Felch, "Effect of the azimuthal inhomogeneity of electron emission on gyrotron operation," Phys. Plasmas, vol. 8, no. 7, pp. 3473-3479, 2001.

[9] T.L. Grimm, K.E. Kreischer, R.J. Temkin, "Experimental study of a megawatt 200-300 GHz gyrotron oscillator," Physics of Fluids B (Plasma-Physics), vol. 5, no. 11, pp. 4135-43, Nov. 1993.

[10] K.A. Bishofberger, "Analysis of azimuthal asymmetries in an annular gyrotron electron beam," Thesis (B.S.), Massachusetts Institute of Technology, Dept. of Physics, 1996 (unpublished).

[11] M. Yu. Glyavin, A. L. Goldenberg, A. N. Kuftin, V. K. Lygin, A. S. Postnikova, and V. E. Zapevalov, "Experimental studies of gyrotron electron beam systems," IEEE Trans. Plasma Sci., vol. 27, no. 2, pp. 474-483, April 1999.

[12] K.E. Kreischer, T. Kimura, B.G. Danly, and R.J. Temkin, "High-power operation of a 170 GHz megawatt gyrotron," Phys. Plasmas, vol. 4, pp. 1907-1914, 1997. 
[13] M. Yu. Glyavin, A. N. Kuftin, N. P. Venediktov, and V. E. Zapevalov, "Experimental investigation of a $110 \mathrm{GHz} / 1 \mathrm{MW}$ gyrotron with the one-step depressed collector," Int. J. Infrared Millimeter Waves, vol. 18, no. 11, pp. 2129-2136, 1997.

[14] G. Dammertz, O. Braz, A.K. Chopra, K. Koppenburg, M. Kuntze, B. Piosczyk, and M. Thumm, "Recent results of the 1-MW, 140-GHz, TE 22,6 -mode gyrotron," IEEE Trans. Plasma Sci., vol. 27, no. 2, pp. 330-339, April 1999.

[15] W. B. Hermannsfeldt. Electron trajectory program. (226), November 1979.

[16] T. Kimura, Experimental study of a $1 \mathrm{MW}, 170 \mathrm{GHz}$ gyrotron oscillator, PhD thesis, Massachusetts Institute of Technology, 1997.

[17] R. T. Longo, E. A. Adler, and L. R. Falce, "Dispenser cathode life prediction model," IEDM Conf. Dig., pp. 318-324, 1984.

[18] G. Eng, "A new correction to Schottky barrier lowering in cathodes," Aerospace Corp. Rep. SD-TR-85-42, July 15, 1985.

[19] R. Vaughan, "A synthesis of the Long and Eng cathode emission models," IEEE Trans. Elec. Dev., vol. ED-33, no. 11, pp. 1925-1927, Nov. 1986.

[20] A. S. Gilmour, Jr., Principles of Traveling Wave Tubes, (Artech House, Norwood, MA, 1994), Ch 5.

[21] J. C. Tonnerre, D. Brion, P. Palluel, and A. M. Schroff, "Evaluation of the work function distribution of impregnated cathodes," Appl. Surf. Sci., vol. 16, pp. 238-249, 1983.

[22] J. W. Gibson, G. A. Haas, and R. E. Thomas, "Investigation of scandate cathodes: emission, fabrication and activation processes," IEEE Trans. on Elec. Dev., vol. 36, no. 1, pp. 209-214, Jan. 1989.

[23] C. M. Fortgang, "Measuring current emission and work functions of large thermionic cathodes," Third IEEE Int'l Vacuum Electronics Conf., IVEC 2002 (Cat. No. 02EX524). IEEE, Piscataway, NJ, USA, pp. 298, 2002.

[24] Sh.E. Tsimring, "Gyrotron electron beams: velocity and energy spread and beam instabilities," Int. J. Infrared Millimeter Waves, vol. 22, no. 10, pp. 1433-1468. 